\title{
Time-of-Flight MR Angiography for Detection of Cerebral Hyperperfusion Syndrome after Superficial Temporal Artery- Middle Cerebral Artery Anastomosis in Moyamoya Disease
}

\author{
(D). Sato, (D) M. Yamada, (DH. Kuroda, (DD. Yamamoto, (D). Asano, (D). Inoue, DK. Fujii, and (D). Kumabe
}

\begin{abstract}
BACKGROUND AND PURPOSE: Cerebral hyperperfusion syndrome is a potential complication of superficial temporal artery-MCA anastomosis for Moyamoya disease. In this study, we evaluated whether TOF-MRA could assess cerebral hyperperfusion syndrome after superficial temporal artery-MCA anastomosis for this disease.
\end{abstract}

MATERIALS AND METHODS: This retrospective study included patients with Moyamoya disease who underwent superficial temporal artery-MCA single anastomosis. TOF-MRA and SPECT were performed before and 1-6 days after anastomosis. Bilateral ROIs on the source image of TOF-MRA were manually placed directly on the parietal branch of the superficial temporal artery just after branching the frontal branch of the superficial temporal artery and on the contralateral superficial temporal artery on the same axial image, respectively. The change ratio of the maximum signal intensity of the superficial temporal artery on TOF-MRA was calculated by using the following formula: (Postoperative Ipsilateral/Postoperative Contralateral)/(Preoperative Ipsilateral/Preoperative Contralateral).

RESULTS: Of 23 patients (26 sides) who underwent the operation, 5 sides showed cerebral hyperperfusion syndrome postoperatively. There was a significant difference in the change ratio of signal intensity on TOF-MRA observed between the cerebral hyperperfusion syndrome and non-cerebral hyperperfusion syndrome groups (cerebral hyperperfusion syndrome group: $1.88 \pm 0.32$; non-cerebral hyperperfusion syndrome group: $1.03 \pm 0.20 ; P=.0009$ ). The minimum ratio value for the cerebral hyperperfusion syndrome group was 1.63 , and the maximum ratio value for the non-cerebral hyperperfusion syndrome group was 1.30 . Thus, no overlap was observed between the 2 groups for the change ratio of signal intensity on TOF-MRA.

CONCLUSIONS: Diagnosis of cerebral hyperperfusion syndrome is indicated by an increase in the change ratio of signal intensity on TOF-MRA by more than approximately 1.5 times the preoperative levels.

ABBREVIATIONS: CHPS = cerebral hyperperfusion syndrome; $\mathrm{MMD}=$ Moyamoya disease; $\mathrm{Obs}=$ observer; $\mathrm{SI}=$ signal intensity; $\mathrm{STA}=$ superficial temporal artery

M oyamoya disease (MMD) is a chronic occlusive cerebrovascular disease characterized by bilateral steno-occlusive changes at the terminal portion of the internal carotid artery and an abnormal vascular network at the base of the brain. ${ }^{1}$ Superficial temporal artery (STA)-MCA anastomosis is the standard surgical treatment for MMD. The incidence of cerebral hyperperfu-

Received September 24, 2015; accepted after revision January 4, 2016.

From the Departments of Neurosurgery (K.S., M.Y., H.K., D.Y., K.F., T.K.) and Diagnostic Radiology (Y.A., Y.I.), Kitasato University School of Medicine, Sagamihara, Kanagawa, Japan.

Dr. Sato conceived the study; Drs Sato, Kuroda, Yamamoto, Inoue, and Asano collected and assembled the data; and Drs Sato, Yamada, and Fujii drafted and Drs Sato, Inoue, and Kumabe co-wrote the manuscript. All authors discussed the manuscript and approved its final form.

Please address correspondence to: Kimitoshi Sato, MD, Department of Neurosurgery, Kitasato University School of Medicine, 1-15-1 Kitasato, Minami-ku, Sagamihara, Kanagawa, 252-0374 Japan; e-mail: kimitoshi@k8.dion.ne.jp

http://dx.doi.org/10.3174/ajnr.A4715 sion syndrome (CHPS) after STA-MCA anastomosis for MMD was reported to range from $15.0 \%$ to $38.2 \% .^{2-5}$ With CHPS, transient focal neurologic deterioration is often exhibited, and if intracerebral hemorrhage occurs, it may lead to permanent neurologic deficits. ${ }^{6}$ Therefore, it is important to evaluate the regional cerebral blood flow after STA-MCA anastomosis for MMD.

Positron-emission tomography and single-photon emission CT are criterion standard tools for the evaluation of regional CBF in patients with $\mathrm{MMD}^{4,7-9}$; however, they are time-consuming modalities and require the injection of a radiotracer. MR imaging is a powerful method for comprehensive assessment of the brain without radiation exposure, and time-of-flight MR angiography is widely available. An increase in the signal intensity (SI) of the donor from the STA on TOF-MRA after STA-MCA anastomosis is observed in some patients with MMD. ${ }^{2,3,6}$ Furthermore, we have previously reported that TOF-MRA revealed an increase in SI at the middle cerebral artery after carotid endarterectomy and 


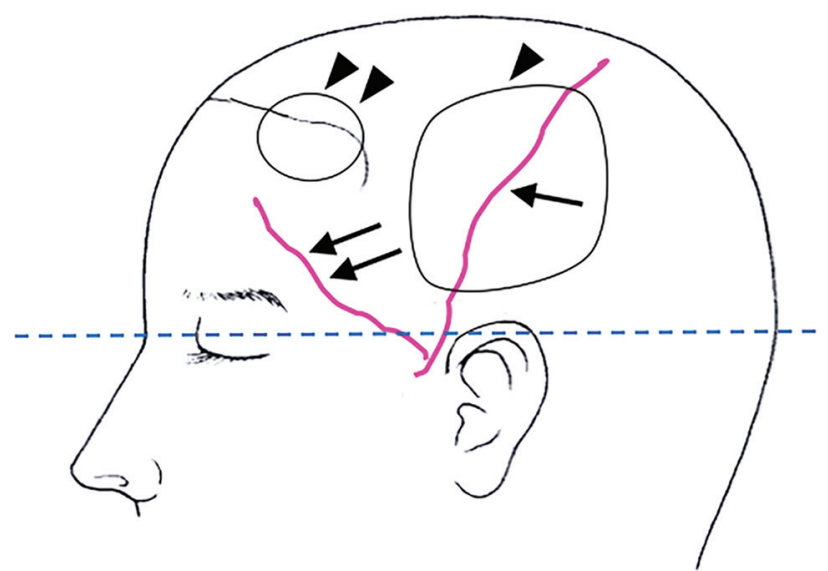

FIG 1. The schema shows the procedure for encephalo-myo-synangiosis (arrowhead) and encephalo-galeo-synangiosis (double arrowheads). The dashed line indicates the estimated axial section of timeof-flight MR angiography on the parietal branch (arrow) of the superficial temporal artery immediately after branching the frontal branch (double arrows) of the STA.

that the SI changes corresponded to acute hemodynamic changes. ${ }^{10}$ In the present study, we used similar methods to retrospectively investigate whether CHPS diagnosis could be performed on the basis of the degree of increased SI in the donor from the parietal branch of the STA on TOF-MRA after STAMCA anastomosis for MMD.

\section{MATERIALS AND METHODS}

The ethics committee of our hospital approved this study, and the requirement for informed consent was waived (B14-229).

\section{Subjects and Surgery}

All patients were diagnosed with MMD according to the criteria issued by the Japanese Ministry of Health, Labor, and Welfare. ${ }^{12}$ The indications for surgical revascularization were patients with ischemic symptoms or hemodynamic compromise as detected by SPECT. The same surgeon (K.S.) performed STA-MCA anastomosis by using a parietal branch of the STA + encephalo-myosynangiosis and encephalo-galeo-synangiosis procedures with the patient under general anesthesia between October 2009 and August 2013 at the Kitasato University Hospital (Fig 1). Of the 35 patients undergoing the MMD operation (41 sides), 12 patients (15 sides) were excluded due to a lack of MRA or SPECT data. The remaining 23 consecutive patients ( 26 sides, 11 patients younger than 16 years of age; 7 male patients, 16 female patients; age range, 6-67 years; mean age, 25 years) were subject to analysis. MR imaging/TOF-MRA and iodine $123 \mathrm{~N}$-isopropyl-p-iodoamphetamine ( ${ }^{123}$ I-IMP)-SPECT evaluation were performed before the operation and within 1-6 days after the operation in all 23 patients.

\section{Assessment of STA Signal Intensity on MRA Images}

A 1.5T scanner (Signa Excite HD or HDxt or CV/i; GE Healthcare, Milwaukee, Wisconsin) was used to perform MRA. Axial TOFMRA images of the intracranial arteries, parallel to the anterior/ posterior commissure line and covering the top of the head, were acquired by using an 8- or 12-channel head coil or a single-channel quadrature head coil (HD or HDxt [TR, 25 ms; TE, 2.7 ms; flip angle, $18^{\circ}$; FOV, $22 \mathrm{~cm}$; matrix, $320 \times 192$; section thickness, 1.6 mm; NEX, 1 acquisition; time, 5 minutes 19 seconds]; CV/i [TR, $25 \mathrm{~ms}$; TE, $6.9 \mathrm{~ms}$; flip angle, $18^{\circ}$; FOV, $22 \mathrm{~cm}$; matrix, $288 \times 160$; section thickness, $1.6 \mathrm{~mm}$; NEX, 1 acquisition; time, 6 minutes 11 seconds]). Bilateral ROIs on the source image of TOF-MRA were manually placed on the parietal branch of the STA just after branching the frontal branch of the STA and on the contralateral STA on the same axial image. The maximum SI was determined for each ROI. Three neurosurgeons (observer [Obs] 1, K.S.; Obs 2, H.K.; and Obs 3, D.Y, with $>10$ years of experience) placed the ROIs independently, and Obs 1 placed the ROI twice at an interval of $>3$ months without knowledge of the clinical information or SPECT findings.

\section{Change Ratio of SI of the STA on MRA}

The ipsilateral and contralateral SI values were used to create postoperative and preoperative ratios.

The ipsilateral-to-contralateral ratio was determined as the ratio of the maximum SI for the ipsilateral ROI and the contralateral ROI. The change ratio of SI was calculated by dividing the postoperative ipsilateral-to-contralateral ratio by the preoperative ipsilateral-to-contralateral ratio. ${ }^{10}$

\section{Definition of CHPS}

The diagnosis of CHPS satisfied all criteria as follows: 1) the presence of focal neurologic signs; 2) confirmed patency of the bypass by MRA and the absence of any ischemic changes by diffusionweighted imaging; 3 ) marked postoperative increase in regional CBF on SPECT in the ipsilateral hemisphere and exceeding that in the contralateral hemisphere; and 4) the absence of other pathologies such as compression of the brain surface by swelling of the temporal muscle graft or ischemic attack. All patients were evaluated by the same clinician for the presence of CHPS.

\section{Statistical Analysis}

Differences between the 2 groups (with or without CHPS) were evaluated by the Mann-Whitney $U$ test for continuous variables (age) and the Fisher exact test (sex, initial symptom at disease onset, and operation side) for absolute categoric variables. We assessed the measures of the reliability of the change ratio of SI of the STA on MRA for continuous measures by using intraclass correlation coefficients. The Mann-Whitney $U$ test was used for comparison in the change ratio of SI between the CHPS and nonCHPS groups. The larger change ratio of SI was analyzed for the 3 patients with bilateral operations, and we used 1 side per patient in the analysis. A $P$ value $<.05$ was statistically significant. All statistical analyses were conducted by using JMP 10 software (SAS Institute, Cary, North Carolina).

\section{RESULTS}

Of 23 patients ( 26 sides) who underwent the operation, 5 patients ( 5 sides) showed CHPS postoperatively. The baseline characteristics of the patients are shown in Table 1. There were no statistically significant differences in age, sex, initial symptom at disease onset (TIA or infarction), and operation side between groups.

The change ratio of SI regarding TOF-MRA was significantly larger in the CHPS group than in the non-CHPS group (CHPS 
Table 1: Clinical baseline characteristics of the patients

\begin{tabular}{lccc}
\hline & CHPS $+(\boldsymbol{n}=5)$ & CHPS- $(\boldsymbol{n}=18)$ & $\boldsymbol{P}$ Value \\
\hline Median age (25\%, 75\%) (yr) & $32.0(16.5,60.0)$ & $15.5(11.8,39.2)$ & $.28^{\mathrm{a}}$ \\
Sex (male/female) & $1: 4$ & $6: 12$ & $1.0^{\mathrm{b}}$ \\
Presentation at onset (TIA/infarction) & $3 / 2$ & $15 / 3$ & $.29^{\mathrm{b}}$ \\
Operation side (right/left) & $3 / 2$ & $9 / 9$ & $1.0^{\mathrm{b}}$ \\
\hline
\end{tabular}

Note:-CHPS+ indicates patients with cerebral hyperperfusion syndrome; CHPS-, patients without cerebral hyperperfusion syndrome.

${ }^{a}$ Mann-Whitney $U$ test

${ }^{b}$ Fisher exact test.

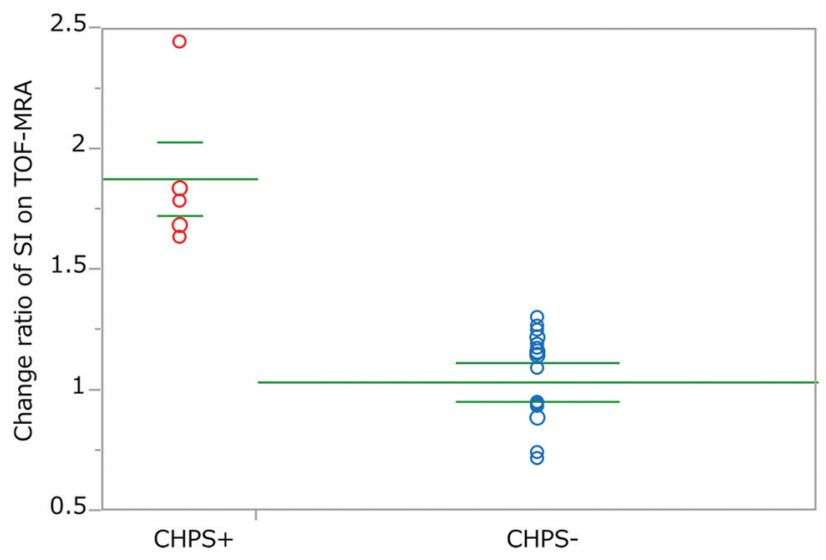

FIG 2. The change ratio of signal intensity on time-of-flight MR angiography observed for the cerebral hyperperfusion syndrome group and the non-CHPS group. The horizontal lines represent the median, first quartile, and third quartile. CHPS + indicates patients with cerebral hyperperfusion syndrome; CHPS-, patients without cerebral hyperperfusion syndrome.

group: $1.88 \pm 0.32$; range, $1.63-2.44$; non-CHPS group: $1.03 \pm$ 0.20 ; range, $0.71-1.30 ; P=.0009$ ) (Fig 2). The minimum ratio value for the CHPS group was 1.63, and the maximum ratio value for the non-CHPS group was 1.32. Thus, no overlap was observed between the 2 groups for the change ratio of SI on TOF-MRA (Table 2).

A summary of the 5 patients with postoperative CHPS is shown in Table 2. All patients immediately recovered after surgery without developing new neurologic deficits. Furthermore, patients did not exhibit additional ischemic lesions on postoperative MR images, including T1-, T2-, and diffusion-weighted images. Postoperative bypass patency was confirmed in all cases.

The reliability of the calculation of the change ratio of SI was assessed by using intraclass correlation coefficients. Strong intraobserver agreement was demonstrated for Obs 1 (intraclass correlation coefficient, $r=0.9830$ ). When the first estimates obtained by Obs 1 were compared with the estimates obtained by Obs 2 and Obs 3, interobserver agreement was also excellent (versus Obs 2: intraclass correlation coefficient, $r=0.9572$; versus Obs 3: intraclass correlation coefficient, $r=0.9641$ ). On the basis of these findings, the first estimates obtained by Obs 1 were used for analysis.

\section{Representative Cases}

Case 1. A 53-year-old woman who had a visual disturbance caused by a right occipital infarction and sensory disturbance in the left hemisphere was diagnosed with MMD (Fig 3). She underwent a bypass operation consisting of right STA-MCA + encephalo-myo-synangiosis and encephalo-galeo-synangiosis. Sub- arachnoid hemorrhage occurred 4 days after the operation, and CHPS was diagnosed. Her change ratio of SI was 2.44 . Subsequently, CHPS disappeared, and the patient was discharged from the hospital without any new neurologic deficit.

Case 2. A 13-year-old boy had experienced TIAs and was diagnosed with MMD (Fig 4). He underwent a bypass operation consisting of left STA-MCA + encephalo-myo-synangiosis and encephalo-galeo-synangiosis. No postoperative CHPS was observed (change ratio of SI $=0.93$ ), and the patient was discharged without a neurologic deficit.

\section{DISCUSSION}

In the present study, we evaluated TOF-MRA findings and postoperative CHPS after STA-MCA anastomosis for MMD. The change ratio of SI in the donor vessel was significantly larger in the CHPS group than in the non-CHPS group, and a cutoff of 1.5 completely discriminated patients with CHPS from those without CHPS. We suggest that MRA can be used to screen patients with CHPS after STA-MCA anastomosis.

\section{CHPS after STA-MCA Anastomosis for Moyamoya Disease}

CHPS following cerebral revascularization is well-recognized, particularly in the context of carotid endarterectomy. In previous reports on carotid endarterectomy, postoperative CHPS was most commonly defined as an increase of $>100 \%$ over baseline (preoperative values). ${ }^{12}$ However, there is no reported definition of CHPS after STA-MCA anastomosis for MMD, to our knowledge.

Increasing evidence suggests that CHPS may occur after a revascularization operation in $15.0 \%-38.2 \%$ of patients with MMD, more frequently than after a revascularization operation for atherosclerotic disease, despite the same STA-MCA bypass operation. $^{2-5}$ In addition, CHPS occurs more frequently in adult MMD than in pediatric $\mathrm{MMD}^{3-5,13}$ and leads to transient neurologic deterioration, seizures, or delayed intracerebral hemorrhage. ${ }^{6}$ Therefore, careful management of CHPS is mandatory after anastomosis for MMD. ${ }^{14}$

\section{Hemodynamic Study in Moyamoya Disease}

Although SPECT, positron-emission tomography, xenonenhanced CT, dynamic perfusion CT, and MR imaging with dynamic susceptibility contrast and arterial spin-labeling have been reported useful for evaluating cerebral hemodynamics in MMD, ${ }^{4,8,9}$ they involve radiation exposure or have limitations in availability. In contrast, TOF-MRA does not require the administration of radioisotopes and contrast agents, can be undertaken quickly, and is widely available.

\section{Underlying Mechanisms of Change of Signal Intensity on TOF-MRA}

TOF-MRA is a widely used noninvasive technique for evaluating intracranial arteries. The SI loss attributable to the saturation effect is flow-velocity-dependent and more pronounced at lower 
Table 2: Summary of 5 patients with CHPS

\begin{tabular}{|c|c|c|c|c|c|c|}
\hline $\begin{array}{l}\text { Age } \\
\text { (yr) }\end{array}$ & Sex & $\begin{array}{l}\text { Initial Presentation } \\
\text { at Disease Onset }\end{array}$ & CHPS Symptom & $\begin{array}{c}\text { Duration from Operation } \\
\text { to CHPS (days) }\end{array}$ & $\begin{array}{l}\text { Duration from Operation } \\
\text { to TOF-MRA (days) }\end{array}$ & $\begin{array}{l}\text { Change Ratio of SI } \\
\text { on TOF-MRA }\end{array}$ \\
\hline 26 & Female & TIA & Numbness of upper limb & 2 & 1 & 1.63 \\
\hline 32 & Female & Infarction & Partial seizure & 3 & 4 & 1.68 \\
\hline 67 & Female & TIA & Palsy of upper limb & 1 & 5 & 1.79 \\
\hline 7 & Male & TIA & $\begin{array}{l}\text { Numbness of upper limb } \\
\text { and dysarthria }\end{array}$ & 5 & 3 & 1.84 \\
\hline 53 & Female & Infarction & Subarachnoid hemorrhage & 4 & 4 & 2.44 \\
\hline
\end{tabular}
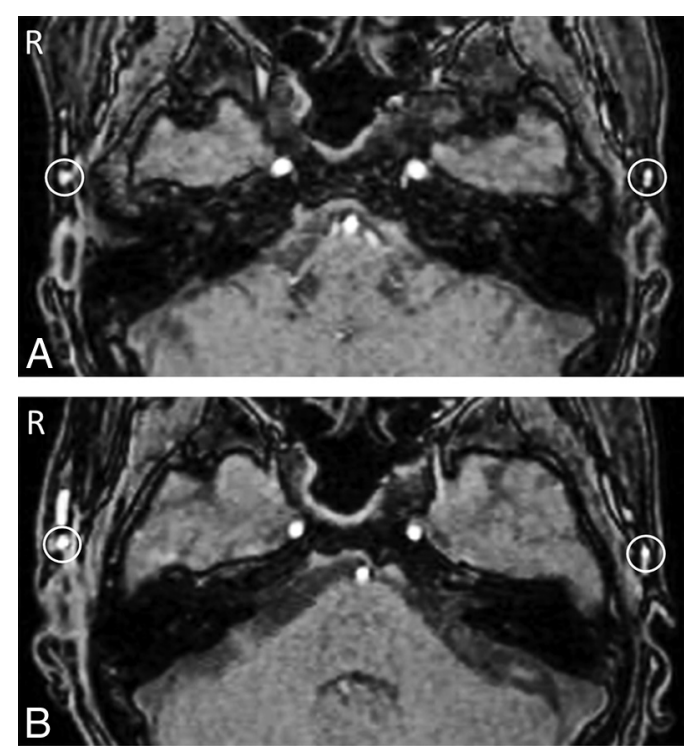

FIG 3. Preoperative $(A)$ and postoperative $(B)$ time-of-flight MR angiography in a case in which cerebral hyperperfusion syndrome was observed after left superficial temporal artery-middle cerebral artery anastomosis. Bilateral regions of interest were manually placed on the parietal branch of the STA immediately after branching the frontal branch of the STA on the same axial image at the axial sections of TOF-MRA; the signal intensity of the graft from the parietal branch of the STA increased postoperatively $(B)$ compared with preoperatively $(A)$.

flow velocities, ${ }^{15}$ particularly on single-slab 3D TOF-MRA. ${ }^{16}$ On the basis of the contrast mechanism of TOF-MRA, which reflects flow-related enhancement, a change in SI on TOF-MRA can partly reflect the hemodynamics of flowing blood. The degree of enhancement in flowing blood on MRA images, referred to as SI within the vessel lumen, increases nonlinearly with increasing absolute flow velocity. ${ }^{17,18}$ MIP images are frequently used for TOFMRA. However, because the target blood vessels in the present study were thin and were depicted as having a low SI, they were difficult to assess on MIP images. Thus, source images were used for assessment. Furthermore, we consider that the use of maximum SI improves reproducibility.

Kohama et al ${ }^{19}$ reported a case with intense high signal of the donor STA and dilated branches of the MCA around the site of anastomosis on 3T MRA, but not 1.5T MRA. However, the results of the present study were acquired by using $1.5 \mathrm{~T}$, proving that these findings were not 3T-specific. Moreover, strong intra- and interobserver agreement was obtained by using our method.

\section{Study Limitations}

We used 3 types of 1.5T MR imaging scanners in this series. Although further studies are required to assess possible scan-
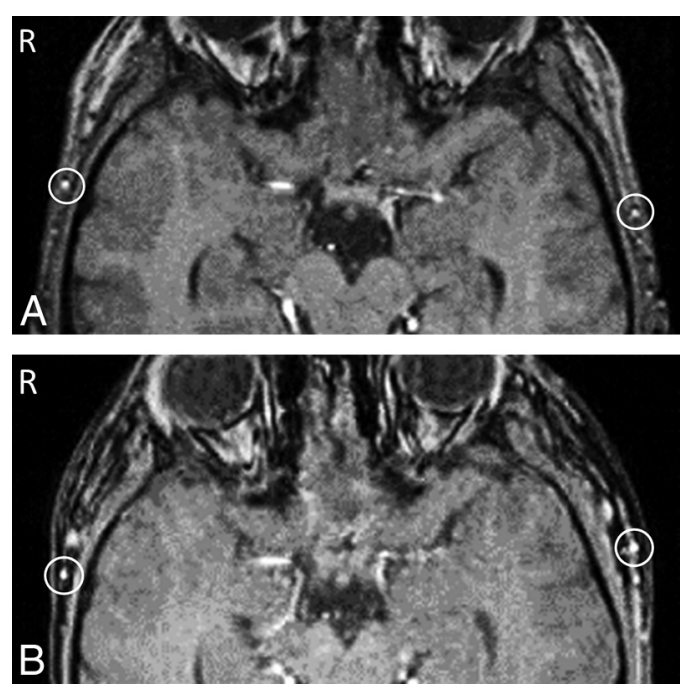

FIG 4. Preoperative $(A)$ and postoperative $(B)$ time-of-flight MR angiography in a case in which cerebral hyperperfusion syndrome was not observed after left superficial temporal artery-middle cerebral artery anastomosis. Bilateral ROIs were manually placed on the parietal branch of the STA immediately after branching the frontal branch of the STA on the same axial image at the axial sections of TOF-MRA; the signal intensity of the graft from the parietal branch of the STA did not increase postoperatively $(B)$ compared with preoperatively $(A)$.

ner-related differences by comparing the increase in the change ratio on both ipsilateral and contralateral sides, our method can help reduce the effects of scanner-related differences and physiologic fluctuations such as blood pressure and partial pressure of carbon dioxide in arterial blood. ${ }^{20,21} \mathrm{Be}$ cause our study included both adults and children, ${ }^{123} \mathrm{I}$-IMPSPECT was used in both a quantitative and qualitative manner. However, because the TOF-MRA method does not require administration of radiotracers or the securing of an intravenous line, this method may be effectively applied to pediatric cases with ease. Uchino et $\mathrm{al}^{5}$ reported that the onset of CHPS varied from 0 to 9 days after the operation (mean, $3.5 \pm 3.5$ days). In the present study, TOF-MRA and ${ }^{123}$ I-IMP-SPECT were not always performed on the same day within 1-6 days after surgery. Because this was a single-institution study with a small sample size, future comprehensive studies with more cases are required.

\section{CONCLUSIONS}

Our results suggest that an increase of the change ratio of SI on TOF-MRA of more than approximately 1.5 times the preoperative levels is useful for screening CHPS. 


\section{ACKNOWLEDGMENTS}

We thank Dr Keika Hoshi, Department of Preventive Medicine, Kitasato University School of Medicine, for data analysis.

Disclosures: Toshihiro Kumabe-UNRELATED: Payment for Lectures (including service on Speakers Bureaus): Eisai.

\section{REFERENCES}

1. Suzuki J, Takaku A. Cerebrovascular "moyamoya” disease: disease showing abnormal net-like vessels in base of brain. Arch Neurol 1969;20:288-99 CrossRef Medline

2. Fujimura $M$, Niizuma $K$, Inoue $T$, et al. Minocycline prevents focal neurological deterioration due to cerebral hyperperfusion after extracranial-intracranial bypass for moyamoya disease. Neurosurgery 2014;74:163-70; discussion 170 CrossRef Medline

3. Fujimura M, Shimizu $H$, Inoue $T$, et al. Significance of focal cerebral hyperperfusion as a cause of transient neurologic deterioration after extracranial-intracranial bypass for moyamoya disease: comparative study with non-moyamoya patients using $\mathrm{N}$-isopropylp-[(123)I]iodoamphetamine single-photon emission computed tomography. Neurosurgery 2011;68:957-64; discussion 964-65 CrossRef Medline

4. Kaku Y, Iihara K, Nakajima N, et al. Cerebral blood flow and metabolism of hyperperfusion after cerebral revascularization in patients with moyamoya disease. J Cereb Blood Flow Metab 2012;32:2066-75 CrossRef Medline

5. Uchino H, Kuroda S, Hirata K, et al. Predictors and clinical features of postoperative hyperperfusion after surgical revascularization for moyamoya disease: a serial single photon emission CT/positron emission tomography study. Stroke 2012;43:2610-16 CrossRef Medline

6. Fujimura M, Shimizu H, Mugikura S, et al. Delayed intracerebral hemorrhage after superficial temporal artery-middle cerebral artery anastomosis in a patient with moyamoya disease: possible involvement of cerebral hyperperfusion and increased vascular permeability. Surg Neurol 2009;71:223-27; discussion 227 CrossRef Medline

7. Horie N, Morikawa M, Morofuji $\mathrm{Y}$, et al. De novo ivy sign indicates postoperative hyperperfusion in moyamoya disease. Stroke 2014; 45:1488-91 CrossRef Medline

8. Lee M, Zaharchuk G, Guzman R, et al. Quantitative hemodynamic studies in moyamoya disease: a review. Neurosurg Focus 2009;26:E5 CrossRef Medline

9. Sugino T, Mikami T, Miyata K, et al. Arterial spin-labeling magnetic resonance imaging after revascularization of moyamoya disease. $J$ Stroke Cerebrovasc Dis 2013;22:811-16 CrossRef Medline

10. Sato K, Kurata A, Oka H, et al. Signal intensity changes for the middle cerebral artery on 3-dimensional time-of-flight magnetic reso- nance angiography indicate acute hemodynamic changes after carotid endarterectomy. J Stroke Cerebrovasc Dis 2013;22:e511-15 CrossRef Medline

11. Research Committee on the Pathology and Treatment of Spontaneous Occlusion of the Circle of Willis; Health Labour Sciences Research Grant for Research on Measures for Intractable Diseases. Guidelines for diagnosis and treatment of moyamoya disease (spontaneous occlusion of the circle of Willis). Neurol Med Chir (Tokyo) 2012;52:245-66 CrossRef Medline

12. Piepgras DG, Morgan MK, Sundt TM Jr, et al. Intracerebral hemorrhage after carotid endarterectomy. J Neurosurg 1988;68:532-36 Medline

13. Fujimura M, Mugikura S, Kaneta $T$, et al. Incidence and risk factors for symptomatic cerebral hyperperfusion after superficial temporal artery-middle cerebral artery anastomosis in patients with moyamoya disease. Surg Neurol 2009;71:442-47 CrossRef Medline

14. Fujimura $\mathrm{M}$, Inoue $\mathrm{T}$, Shimizu $\mathrm{H}$, et al. Efficacy of prophylactic blood pressure lowering according to a standardized postoperative management protocol to prevent symptomatic cerebral hyperperfusion after direct revascularization surgery for moyamoya disease. Cerebrovasc Dis 2012;33:436-45 CrossRef Medline

15. Kodama $T$, Watanabe $K$. Influence of imaging parameters, flow velocity, and pulsatile flow on three-dimensional time-of-flight MR angiography: experimental studies. Eur J Radiol 1997;26:83-91 CrossRef Medline

16. Davis WL, Blatter DD, Harnsberger $\mathrm{HR}$, et al. Intracranial MR angiography: comparison of single-volume three-dimensional time-of-flight and multiple overlapping thin slab acquisition techniques. AJR Am J Roentgenol 1994;163:915-20 CrossRef Medline

17. Leng X, Ip HL, Soo Y, et al. Interobserver reproducibility of signal intensity ratio on magnetic resonance angiography for hemodynamic impact of intracranial atherosclerosis. J Stroke Cerebrovasc Dis 2013;22:e615-19 CrossRef Medline

18. Leng X, Wong KS, Soo Y, et al. Magnetic resonance angiography signal intensity as a marker of hemodynamic impairment in intracranial arterial stenosis. PLoS One 2013;8:e80124 CrossRef Medline

19. Kohama M, Fujimura M, Mugikura S, et al. Temporal change of 3-T magnetic resonance imaging/angiography during symptomatic cerebral hyperperfusion following superficial temporal arterymiddle cerebral artery anastomosis in a patient with adult-onset moyamoya disease. Neurosurg Rev 2008;31:451-55; discussion 455 CrossRef Medline

20. Greenberg JH, Kushner M, Rango M, et al. Validation studies of iodine-123-iodoamphetamine as a cerebral blood flow tracer using emission tomography. J Nucl Med 1990;31:1364-69 Medline

21. Naylor AR, Merrick MV, Slattery JM, et al. Parametric imaging of cerebral vascular reserve, 2: reproducibility, response to $\mathrm{CO}_{2}$ and correlation with middle cerebral artery velocities. Eur J Nucl Med 1991;18:259-64 Medline 